\title{
Interparlamentarische Demokratie? \\ Zur Einbindung der nationalen Parlamente in die Rechtsetzung der Europäischen Union
}

\author{
Mathias Rossi
}

\section{A. Konzept der demokratischen Legitimation der Europäischen Union}

Wegen des Anwendungsvorrangs des Rechts der Europäischen Union vor nationalem Recht und mit zunehmender Kompetenzübertragung von den Mitgliedstaaten auf dic Europäische Union durch die zahlreichen Vertragsänderungen seit der Einheitlichen Europäischen Akte ist das Bewusstsein für die demokratische Legitimationsbedürftigkeit der europäischen Rechtsetzung kontinuierlich geschärft worden. Die - im Ergebnis nicht zutreffende - These vom Demokratiedefizit der Europäischen Union hält sich beharrlich und bestimmt jede oberflächliche Diskussion über den Sinn und die Befugnisse der Europäischen Union. Insofern ist es nicht verwunderlich, dass der Vertrag von Lissabon - stärker noch als der Entwurf über einen Verfassungsvertrag - das Konzept der demokratischen Legitimation in den Art. 10 - 12 EUV explizit offen legt. Neben dem in Art. 10 EUV festgeschriebenen Grundsatz der repräsentativen Demokratie (I.) sind dabei mit Art. 11 EUV erstmals Elemente partizipativer Demokratie (II.) und in Art. 12 EUV zudem Aspekte interparlamentarischer Demokratie (III.) in das Primärrecht aufgenommen worden. Nach einer kurzen Rekapitulation der ersten beiden Legitimationsformen soll die dritte demokratische Erscheinungsform, die interparlamentarische Demokratie, näher betrachtet werden. Dass dabei im Ergebnis insgesamt eine gewisse Skepsis durchschlägt, ist nicht zuletzt auch dem ebenso unvoreingenommenen wie kritischen Blick Thilo Brandners geschuldet, mit dem er das Europarecht und seine rasante Entwicklung in den letzten 25 Jahren betrachtet hat.

- Meinem Mitarbeiter, Herm Christion Hufen, danke ich vielmals für die wertvolle Unterstützung. 


\section{Grundsatz repräsentativer Demokratie}

Art. 10 EUV hebt zunächst den Grundsatz der repräsentativen Demokratie hervor. Während Abs. 1 diesen einerseits ganz konkret, andererseits aber auch konkretisierungsbedürftig benennt, formuliert Abs. 2 die beiden Legitimationsstränge, über die die beiden zu legitimierenden Rechtsetzungsorgane mit den Legitimationssubjekten verbunden sind: Das Europäische Parlament wird unmittelbar demokratisch legitimiert durch die Bürgerinnen und Bürger, der Europäische Rat und der Rat der Europäischen Union werden mittelbar demokratisch legitimiert durch die in ihnen vertretenen Staats- und Regierungschefs bzw. die jeweiligen Regierungsvertreter.' Drei Aspekte seinen dabei in diesem Kontext kurz hervorgehoben:

Zunächst sei in Erinnerung gerufen, dass die demokratische Legitimation der Europäischen Union respektive der Europäischen Gemeinschaften ursprünglich ganz überwiegend über den Rat erfolgte. Dies war insofem konsequent, als nur dieser verbindliche Rechtsetzungskompetenzen innehatte. Erst mit der zunehmenden Befugniserweiterung des Europäischen Parlaments wuchs auch die Notwendigkeit seiner stärkeren demokratischen Legitimation. Die Einführung der Direktwahlen des Europåischen Parlaments und sein ständiger Zuwachs an Einflussmöglichkeiten auf die Rechtsetzung und den Haushalt der EU stehen somit in einem wechselseitigen, spiralähnlichen Zusammenspiel: Die Befugniserweiterung des Parlaments verlangt nach einer stärkeren Legitimation, die ihrerseits stärkere Befugnisse verlangt. Vor diesem Hintergrund ist es auch die unter der Flagge des Demokratieprinzips artikulierte Forderung nach weiteren Befugnissen des Europäischen Parlaments, die die Europäische Union in die Nähe einer Staatlichkeit führt, die letztlich doch nicht gewollt ist.

Diese Entwicklung muss deshalb betont werden, weil sie der Systematik des Art. 10 Abs. 2 EUV nicht mehr zu entnehmen ist: Wegen der (vermeintlich) höheren Bedeutung der unmittelbaren Legitimation des Europäischen Parlaments wird dieser Strang als erster genannt, wohingegen sich die über den Europäischen Rat und den Rat der EU vermitteltc Legitimation erst an zweiter Stelle befindet. Verstärkt wird die Hervorhebung des Europäischen Parlaments zusätzlich dadurch, dass als Legitimationssubjekte nicht mehr die „Völker der Mitgliedstaaten“, sondern die „Bürgerinnen und Bürger“ genannt sind. Damit soll wohl unabhängig nationalstaatlicher Gliederungen unmittelbar an die Unionsbürger angeknüpft werden, was mindestens in zweifacher Hinsicht widersprüchlich bleibt: Erstens wird die Unionsbürgerschaft streng akzessorisch aus einer mitglied- 
staatlichen Staatsangebörigkeit abgeleitet, und zweitens und vor allem ist auch das Wahlrecht in Bezug auf das Europäische Parlament nach wie vor mitgliedstaatlich ausgestaltet. Vor diesem Hintergrund und angesichts der mitgliedstaatlichen Kontingentierung der Sitze im Europäischen Parlament kann jedenfalls die Gleichheit der Wahl nicht unionsweit gewährleistet werden - ein Umstand, dem das Primärrecht dadurch Rechnung trägt, dass es die Gleichheit gar nicht erst als Wahlrechtsgrundsatz verlangt. Konsequenterweise vermeiden Art. 10 Abs. 2 EUV wie auch Art. 14 Abs. 2 EUV es, von den Mitgliedern des Europäischen Parlaments als Vertreter des (also eines) Europäischen Volks zu sprechen.

Die (theoriegeschaffenen) Problerne der Bestimmung des Legitimationssubjekts bzw. der Legitimationssubjekte der Europäischen Union im Allgemeinen und des Europäischen Parlaments im Besonderen werden weitgehend irrelevant, wenn man sich mit Art. 10 Abs. 2 UAbs. 2 EUV darauf besinnt, dass die Europäische Union von einem Zwittercharakter ist: Sie ist ein Zusammenschluss der Staatsbürger in den Mitgliedstaaten, die in ihrer Summe als Unionsbürger bezeichnet werden mögen, und zugleich ein Zusammenschluss der Mitgliedstaaten selbst. Die Betonung dieser ursprünglich angelegten Architektur der Europäischen Union hilft auch zu vermeiden, die - überflüssige - Diskussion über die Frage der Finalität der Europäischen Union durch eine staatstheoretische Brille zu führen. Die Europäische Union sollte stets ein Gebilde sui generis sein und ist es auch heute noch. Deshalb kann und darf sie auch auf besondere Art und Weise demokratisch legitimiert werden.

\section{Elemente partizipativer Dennokratie}

Zu der besonderen Art und Weise der demokratischen Legitimation sollen nach Art. 11 EUV zusätzlich Elemente partizipativer Demokratie beitragen, die hier nur genannt seien:

Wenn Art. 11 Abs. 1 EUV auch vollmundig davon spricht, dass „die Otgane den Bürgerinnen und Bürgern in geeigneter Weise die Möglichkeit geben, ihre Ansichten in allen Bereichen des Handelns der Union öffentlich bekannt zu geben und auszutauschen," verbirgt sich bei normativer Betrachtung doch nicht viel mehr dahinter als die Gewährleistung der Kommunikationsfreiheiten, insbesondere der Meinungsfreiheit, die in der Europäischen Union nicht nur eine Selbstverständlichkeit, sondern auch durch Art. 11 und Art. 12 GRCh verbindlich vorgesehen ist. Die besondere, über diese grundrechtliche Dimension hinausgehende Bedeutung des Art. 11 Abs. 1 EUV liegt deshalb wohl eher in der Einbezie- 
hung der „reprāsentativen Verbände“ in die Gewährung der Rechte, womit bei praktischer Betrachtung einer kollektiven Interessenwahmehmung und bei normativer Betrachtung der Möglichkeit zur kollektiven Grundrechtsausübung Rechnung getragen wird.

Hieran knüpft auch Art. 11 Abs. 2 EUV an, der - ausgesprochen vage - erneut von „den repräsentativen Verbänden“ sowie dann - noch unbestimmter - von „der Zivilgesellschaft" spricht, mit denen die Organe einen „offenen, transparenten und regelmäßigen Dialog“" zu pflegen verpflichtet werden.

Etwas konkreter schreibt Art. 11 Abs. 3 EUV primärrechtlich vor, dass die Europäische Kommission, die von diesem Absatz alleine in die Pflicht genommen wird, ,umfangreiche Anhörungen der Betroffenen" durchzuführen hat. Mit dieser Betroffenenbeteiligung in einer frühen Verfahrensphase der Rechtserzeugung soll „die Kohärenz und die Transparenz des Handelns der Union“" gewährleistet werden, wie die Norm hervorhebt. Von diesen Zielen abgesehen kann die Betroffenenbeteiligung im Rechtsetzungsverfahren die Chance zur Einbeziehung von Sachverstand ebenso bieten wie die Gefahr der Privilegierung bestimmter Gruppen und damit des Verlusts der Allgemeinverbindlichkeit bergen.

Besonderer Ausdruck einer direkt-demokratischen Ausgestaltung des Konzepts demokratischer Legitimation ist schließlich die erstmals im Primärrecht vorgesehene Möglichkeit einer Bürgerinitiative, mit der eine Million Unionsbürger die Europäische Kommission zwar nicht verpflichten, aber immerhin doch auffordern können, einen Rechtsetzungsvorschlag zu unterbreiten ein - Recht, das durch die Verordnung 211/2011 vom 16. 2. 2011 jüngst konkretisiert wurde.

\section{Aspekte interparlamentarischer Demokratie}

Neben diesen Elementen partizipativer und direkter Demokratie wird der Grundsatz der repräsentativen Demokratie von Art. 12 EUV auch um Aspekte einer interparlamentarischen Demokratie ergänzt. Mit dieser Bestimmung werden erstmals primårrechtlich die verschiedenen Einwirkungsmöglichkeiten der nationalen Parlamente auf die europäische Rechtsetzung zusammengefasst, die sich in den letzten vier Jahrzehnten herausentwickelt haben. ${ }^{2}$ Konzeptuell knüpfen sie an eine Verknüpfung der beiden Legitimationsstränge an. indem sie die unmittelbare demokratische Legitimation des Europäischen Parlaments mit der mittelRn. 3 ff. 
baren, nämlich über die nationalen Parlamente vermittelten demokratischen Legitimation des Rats der EU zu verbinden versuchen.

Wie dies im Einzelnen ausgestaltet ist, soll zunächst dargestellt (B.) und sodann zusammenfassend beurteilt werden (C.).

\section{B. Formen der Einbindung der nationalen Parlamente in die Rechtsetzung}

Art. 12 EUV gestaltet insgesamt sechs Formen der Einbindung der nationalen Parlamente in die Beschlussfassung der Europäischen Union aus. Er răumt ihnen ganz allgemein Informationrechte ein ( 1. ) und bindet sie zudem in die Subsidiaritätskontrolle ein (2.). Darüber hinaus stattet er sie bezüglich Maßnahmen auf dem Gebiet des Raums der Freiheit, der Sicherheit und des Rechts (3.) sowie im Vertragsänderungsverfahren (4.) mit besonderen Beteiligungsrechten aus. Auch in das Beitrittsverfahren von beitrittswilligen Drittstaaten sind die nationalen Parlamente eingebunden (5.). Schließlich ist eine interparlamentarische Zusammenarbeit vorgesehenen (6.).

Wenn diese verschiedenen Erscheinungsformen näher betrachtet werden sollen, muss hervorgehoben werden, dass Art. 12 EUV nicht die einzige Rechtsgrundlage der interparlamentarischen Zusammenarbeit ist. Vielmehr sind auf europäischer Ebene zusätzlich die im Protokoll über die Rolle der nationalen Parlamente in der Europäischen Union ${ }^{3}$ sowie im Protokoll über die Anwendung der Grundsätze der Subsidiarität und der VerhältnismäBigkeit ${ }^{4}$ vorgesehenen Verfahren zu beachten und auf nationaler Ebene das Integrationsverantwortungsgesetz (IntVG), das Gesetz über die Zusammenarbeit von Bundesregierung und Deutschem Bundestag in Angelegenheiten der Europaischen Union (EUZBBG) sowie die Geschäftsordnungen des Deutschen Bundestages (GOBT) und des Bundesrates (GOBR) zu berücksichtigen. Ausgehend von der Systematik des Art. 12 EUV werden im Folgenden zunächst die europarechtlichen Vorgaben skizziert und sodann die jeweiligen deutschen Konkretisierungen prässentiert.

\section{Informationsrechte}

Art. 12 lit. a EUV betrifft zunächst die Einbindung der nationalen Parlamente in den allgemeinen Rechtssetzungsprozess der Union. 


\section{Unionsrechtliche Vorgaben}

Die Vorschrift sieht vor, dass die Parlamente der Mitgliedstaaten von den Unionsorganen unterrichtet werden und ihnen die Entwürfe von Gesetzgebungsakten der Union direkt zugeleitet werden. Genaueres hierzu ist in dem Protokoll über die Rolle der nationalen Parlamente in der Europäischen Union geregelt. Unabhängig dieser Konkretisierung liegt die besondere Bedeutung des Art. 12 lit. a EUV darin, dass die nationalen Parlamente unmittelbar von den Unionsorganen und nicht nur mittelbar durch die mitgliedstaatlichen Regierungen über Gesetzgebungsakte informiert werden.

Hinsichtlich des Zeitpunkts der Unterrichtung präzisiert Art. 2 des Protokolls, dass Gesetzgebungsentwürfe der Kommission den nationalen Parlamenten zur gleichen Zeit wie dem Europäischen Parlament zugeleitet werden. Die vom Europäischen Parlament vorgelegten Entwürfe werden den Parlamenten der Mitgliedstaaten direkt zugeleitet. Dabei sind mit „Entwurf“ alle Vorschläge, Äußerungen und Initiativen aller Unionsorgane gemeint, die den Erlass eines Gesetzgebungsaktes zum Ziel haben. Gemäß Art. 4 des Protokolls müssen zwischen dem Zeitpunkt der Zuleitung und dem Zeitpunkt, an dem der Entwurf zwecks Erlass oder zur Festlegung eines Standpunkts im Rahmen eines Gesetzgebungsverfahrens auf die vorläufige Tagesordnung des Rates gesetzt wird, acht Wochen liegen. Hierdurch sollen die nationalen Parlamente die Gelegenheit erhalten, die Entwürfe ausreichend zu prüfen. Weiterhin verlangt Art. 5 des Protokolls, dass die Parlamente auch über die Tagesordnungen sowie die Ergebnisse der Ratstagungen unterrichtet werden.

Diesen relativ umfassenden aktiven Unterrichtungspflichten der europäischen Organe und den mit ihnen korrespondierenden passiven Informationsrechten der nationalen Parlamente stehen nur eingeschränkt Möglichkeiten gegenüber, das europäische Normsetzungsverfahren aktiv, etwa durch Anregungen an die Organe der Europäischen Union, zu beeinflussen. Lediglich bezüglich des Subsidiaritätsprinzips sowie der Verhältnismäßigkeit können die mitgliedstaatlichen Parlamente nach Art. 3 des Protokolls Stellungnahmen an die Präsidenten von Rat, Kommission oder Parlament richten und eine Subsidiaritätsrüge erheben, was in der Sache dadurch gerechtfertigt ist, dass hier die mitgliedstaatlichen Interessen generell und speziell die parlamentarischen Interessen betroffen sind (s. dazu II.). Die Einzelheiten vollziehen sich hierbei nach Maßgabe des Protokolls über die Anwendung der Grundsätze der Subsidiarität sowie der Verhältnismäßigkeit. ${ }^{5}$ Im 
Übrigen sollen die Informationsrechte die nationalen Parlamente lediglich in die Lage versetzen, sich frühzeitig eine eigene Meinung zu dem Gesetzgebungsvorhaben zu bilden und diese gegebenenfalls über die jeweilige Regierung in den Rat der Europäischen Union zu tragen.

Diese in der Gewährung bloßer passiver Informationsrechte zu erkennende Zurückhaltung gegenüber aktiven Einwirkungsmöglichkeiten indiziert, dass Art. 12 EUV zwar einerseits die demokratische Legitimation des Handelns der EU stärken will, andererseits die nationalen Parlamente aber nicht neben den Rechtssetzungsorganen der Union in die Funktion eines Nebengesetzgebers versetzen will. ${ }^{6}$ Im allgemeinen Rechtsetzungsprozess kommt den nationalen Parlamenten also primär eine beobachtende Rolle zu; eine unmittelbare Einflussnahmemöglichkeit auf den Inhait der zu erlassenden Normen besteht nicht.

\section{Innerstaatliche Ausgestaltung}

Über Art. 12 EUV hinaus steht es den Mitgliedstaaten frei, ihre Parlamente in noch höherem Maße an Entscheidungen, die die Mitwirkung des jeweiligen Mitgliedstaates in der Europäischen Union betreffen, zu beteiligen. Neben die Unterrichtung durch die Organe der Europäischen Union kann auf diese Weise eine Unterrichtung durch die jeweilige nationale Regienung treten. In Deutschland ist die Unterrichtung von Bundestag und Bundesrat im Zusammenhang mit Rechtsetzungsakten der Europäischen Union in Art. 23 Abs. 2 S. 2 und Abs. 3 GG festgeschrieben und durch das EUZBBG näher ausgestaltet.

\section{a) Unterrichtung des Bundestages}

Nach Art. 23 Abs. 2 S. 2 GG i.V.m. § 4 EUZBBG unterrichtet die Bundesregierung den Bundestag umfassend und zum frühestmöglichen Zeitpunkt über Vorhaben der Europäischen Union, zu denen nach \$ 3 Abs. 1 Nr. 3 EUZBBG unter anderem auch Vorschläge für Gesetzgebungsakte der EU gehören. Da der Anwendungsbereich des $\$ 3$ Abs. 1 EUZBBG sehr viele Handlungsformen der Organe der EU umfasst - auch der weite Katalog in dieser Vorschrift ist nicht abschließend - und S. 2 lediglich für die GASP eine Bereichsausnahme trifft, gelten dic nachfolgend skizzierten Unterrichtungsgrundsätze auch für đie übrigen von Art. 12 EUV erfassten Bereiche, die eine Mitwirkung der nationalen Parla- 
mente vorsehen. Die Informationspflicht bezieht sich nicht nur auf Entwürfe von Rechtssetzungsakten, sondern erstreckt sich gemäß $\S 3$ Abs. 1 EUZBBG zum Beispiel auch allgemein auf den Bereich der Haushalts- und Finanzplanung oder politische Programme und Aktionspläne der europäischen Organe und erfasst gemäß $\S 4$ Abs. 4 EUZBBG bspw. geplante Rechtsbehelfe der EU vor dem EuGH.

Auch die Art der Informierung ist näher spezifiziert: Dem Bundestag sind durch die Bundesregierung nach $\S 5$ EUZBBG Dokumente und Berichte der europäischen Organe zuzuleiten. Auch hier ist der Umfang der erfassten Dokumente sehr weit und erstreckt sich unter anderem auch auf inoffizielle Dokumente (nonpapers) des Rates und der Kommission. Zusätzlich stellt die Bundesregierung bei Rechtsetzungsvorhaben der Europäischen Union gemäß § 7 Abs. 1 EUZBBG einen Berichtsbogen her, mit dem sie insbesondere eine Bewertung des Vorhabens hinsichtlich seiner Vereinbarkeit mit den Grundsätzen der Subsidiarität und der Verhältnismäßigkeit abgibt. Diese Vorschrift soll dem Bundestag die Abgabe einer Stellungnahme nach Art. 3 des Protokolls über die Rolle der nationalen Parlamente in der Europäischen Union ermöglichen. Darüber hinaus gibt die Bundesregierung nach $\S 7$ Abs. 2 EUZBBG eine umfassende Bewertung ab, die neben den Einschätzungen zu den Grundsätzen der Subsidiarität und der Verhältnismäßigkeit auch solche zu Zuständigkeitsfragen, Umsetzungsbedarf oder Folgen für die Bundesrepublik Deutschland in rechtlicher, wirtschaftlicher oder sozialer Hinsicht enthalten. Hiermit wird dem Bundestag bei der Einschätzung hinsichtlich der Erhebung einer Subsidiaritätsrüge (dazu unten II.) geholfen.

Die Unterrichtung des Bundestages ist also gesetzlich weit ausgestaltet und geht im Umfang über das hinaus, was Art. 12 lit. a ELV von den Unionsorganen verlangt. So sieht $\S 8$ EUZBBG Informations- und Berichtspflichten (einschließlich eines Berichtsbogens zu den Grundsätzen der Subsidiarität und der Verhältnismäßigkeit) der Bundesregierung gegenüber dem Bundestag auf dem Gebiet der GASP vor. Zwar ist dieser Bereich nach $\S 3$ Abs. 1 S. 2 EUZBBG vom Anwendungsbereich der $\S \S 3 \mathrm{ff}$. EUZBBG ausgeschlossen, jedoch sieht $\S 8$ EUZBBG Informationspflichten in etwas eingeschränkterem Umfang vor. Nicht ausdrücklich erfasst von $\S 8$ EUZBBG ist das Recht auf Stellungnahme aus $\S 9$ EUZBBG. Systematische Erwägungen sprechen dafür, dass der Bundestag auch im Bereich der GASP ein Recht auf Stellungnahme hat, da sich die Vorschrift, die das Stellungnahmerecht gewährt, direkt an die über die GASP anschließt. ${ }^{7}$ 
Neben diesen weitgehenden Informationspflichten sind auch die die dem Bundestag im EUZBBG eingeräumten Mitwirkungsrechte weiter, als Art. 12 EUV dies von den Unionsorganen fordert. Nach Art. 23 Abs. 3 S. 1 GG i.V.m. $\$ 9$ Abs. 1 EUZBBG kann der Bundestag Stellungnahmen zu jedem Vorhaben abgeben, also nicht nur zu den Grundsätzen der Subsidiarität und der VerhältnismäBigkeit in Zusammenhang mit Rechtsetzungsakten. Darüber hinaus muss die Bundesregierung bei allen Vorhaben auf Ebene der Europäischen Union die Stellungnahme des Bundestages nach Art. 23 Abs. 3 S. 2 GG berücksichtigen brw. nach $\$ 9$ Abs. 2 EUZBBG ihren Verhandlungen zugrunde legen. Dass der Wortlaut des EUZBBG weiter ist als der des GG ist unbedenklich, da eine Bindung der Bundesregierung über den Grundgesetzwortlaut zulässig ist, solange diese sich innerhalb des Gestaltungsspielraumes der Unionsverträge und des Grundgesetzes befindet. ${ }^{8}$

\$ 9 Abs. 4 S. 1 EUZBBG sieht des Weiteren vor, dass die Bundesregierung einen Parlamentsvorbehalt im Rat einzulegen hat, wenn der Beschluss des Bundestages hinsichtlich eines Rechtsetzungsakts der EU in einem seiner wesentlichen Belange nicht durchsetzbar ist. Dies bedeutet. dass die Bundesregierung sich im Rat noch nicht auf eine verbindliche Position festlegen darf. ${ }^{9}$ Vielmehr soll die Bundesregierung ein Einvernehmen mit dem Bundestag anstreben, wie $\S 9$ Abs. 4 S. 4 EUZBBG es verlangt. Nach $\$ 9$ Abs. 4 S. 6 EUZBBG darf die Bundesregierung aus wichtigen außen- oder integrationspolitischen Gründen von der Stellungnahme des Bundestages abweichen. Daraus ergibt sich aber, dass eine Abweichung nur aus diesen wichtigen Gründen zulässig ist, die Mitwirkungsrechte des Parlaments im Bereich der europäischen Rechtsetzung also stärker ausgeprägt sind, als dies unionsrechtlich erforderlich wäre. Fraglich ist, ob diese $\mathbf{A b}$ weichungseinschränkung sich auch auf Fälle bezieht, in denen die Bundesregierung kein Einvernehmen nach $\S 9$ Abs. 4 S. 4 EUZBBG herstellen kann. Dann müsste die Bundesregierung in diesem Fall grundsätzlich der Stellungnahme des Bundestages folgen. Dagegen spricht aber vor allem der Wortlaut des Art. 23 Abs. 3 GG, so dass $\$ 9$ Abs. 4 EUZBBG nur ein Bemühen der Bundesregierung postuliert, ${ }^{\text {llt }}$ keinesfalls aber eine strikte Bindung der Bundesregierung an die Bundestagsbeschlüsse statuiert. Eine Aushöhlung der der Bundesregierung in

8 Calliess, ZG 2010. I. 24; aA Baach, Parlamentarische Mitwirkung in Angelegenheiten der Europäischen Union, 2008, S. 217 mwN zu beiden Ansichten.

9 Calliess, ZG 2010, 1, 25.

10 Calliess, ZG 2010, 1, 25. 
Art. 23 Abs. 3 S. 2 GG verfassungsrechtlich zugewiesenen Kompetenz ist bei dieser Auslegung des gesetzlichen $§ 9$ EUZBBG nicht zu befürchten. ${ }^{.1}$

b) Unterrichtung des Bundesrates

Die Belange der Bundesländer werden nach Maßgabe des Art. 23 Abs. 4 -6 GG berücksichtigt, die im Gesetz über die Zusammenarbeit von Bund und Ländern in Angelegenheiten der Europäischen Union (EUZBLG) konkretisiert werden. Art. 23 Abs. 4 - 6 GG wie auch das EUZBLG machen den Grad der Einbeziehung des Bundesrates als Vertretungsorgan der Länder auf Bundesebene davon abhängig, ob und welche Länderkompetenzen betroffen sind.

Grundsätzlich wird der Bundesrat nach § 2 EUZBLG über alle Vorhaben der EU unterrichtet, die für Länder von Interesse sein könnten. Nach § 3 EUZBLG hat der Bundesrat bei jedem Vorhaben der EU Gelegenheit zur Stellungnahme, soweit Länderinteressen berührt sind. $\mathrm{Da}$ der Anwendungsbereich dieser Norm auch sehr weit ist, beziehen sich die Ausführungen zur Unterrichtung des Bundestages auch grundsätzlich auf alle von Art. 12 EUV erfassten Mitwirkungsformen. Bei einer Mitwirkungspflicht des Bundesrates oder bei einer Zuständigkeit der Länder muss nach $\S 4$ Abs. 1 EUZBLG ein vom Bundesrat benannter Ländervertreter zu den Beratungen zur Festlegung der Verhandlungsposition hinzugezogen werden. Soweit im Bereich der ausschließlichen Bundeskompetenz Länderinteressen berührt sind oder der Bund ansonsten zur Gesetzgebung befugt ist, wird die Stellungnahme des Bundesrates bei der Festlegung der Verhandlungsposition nach $\S 5$ Abs. 1 EUZBLG berücksichtigt. Wenn bei einem Vorhaben schwerpunktmäßig ausschließliche Gesetzgebungsbefugnisse der Länder betroffen sind, ist die Stellungnahme des Bundesrates schon maßgeblich zu berücksichtigen ( $\$ 5$ Abs. 2 S. 1 EUZBLG), d.h. die Bundesregierung ist grundsätzlich an die Auffassung des Bundesrates gebunden und kann nur in Ausnahmefällen, etwa zur Wahrung der gesamtstaatlichen Verantwortung, davon abweichen. ${ }^{12}$ Sind ausschließliche Gesetzgebungskompetenzen der Länder auf Gebieten der schulischen Bildung, der Kultur und des Rundfunks betroffen, so muss die Bundesregierung die Verhandlungsführung auf europäischer Ebene wegen der Kulturhoheit der Länder einem Ländervertreter übertragen ( $\$ 6$ Abs. 2 S. 1 EUZBLG). Gemäß $\S 6$ Abs. 1 EUZBLG ist der angesprochene Ländervertreter

11 AA Baach, Parlamentarische Mitwirkung in Angelegenheiten der Europäischen Union, 2008, S. 217.

12 Baach. Parlamentarische Mitwirkung in Angelegenheiten der Europäischen Union, 2008. S. 233. 
auch bei den Beratungen innerhalb von Rat und Kommission hinzuzuziehen, wenn der Bundesrat innerstaatlich mitwirkungspflichtig wäre, die Länder zuständig wären oder ihre Interessen berührt würden. Die Einwirkungsmöglichkejten des Ländervertreters auf europäischer Ebene sind allerdings in diesem Verfahren sehr begrenzt, da nach $§ 6$ Abs. 1 S. 2 EUZBLG die Verhandlungsführung bei der Bundesregierung verbleibt und der Vertreter der Länder nur mit ihrem Einverständnis Erklärungen abgeben darf. Diese Regelungen tragen freilich den Besonderheiten des dẹtschen Föderalismus und den Interessen der Länder eher Rechnung als dem Gedanken der parlamentarischen Mitwirkung.

\section{c) Integrationsverantwortung von Bundestag und Bundesrat}

Des Weiteren ist die Mitwirkung von Bundestag und Bundesrat im Integrationsverantwortungsgesetz (IntVG) geregelt, das als Reaktion auf das Urteil des Bundesverfassungsgerichts zum Vertrag von Lissabon erlassen wurde. Dieses Gesetz enthält nicht nur, wie der Name dies suggerieren würde, Pflichten der Gesetzgebungsorgane hinsichtlich ihrer Mitwirkung an der europäischen Integration, sondern auch maßgeblich Rechte und Mitwirkungsbefugnisse. die über die an dieser Stelle dargestellte bloße Unterrichtung hinausgehen. So bedarf es nach $\S 3$ Abs. 1 IntVG etwa eines Gesetzes nach Art. 23 Abs. 1 GG als innerstaatliche Grundlage für die Zustimmung des Bundeskanzlers als Vertreter der Bundesrepublik im Europäischen Rat zu einem Eigenmittelbeschluss nach Art. 311 Abs. 3 AEUV. Dieser Eigenmittelbeschluss wird aufgrund seiner weit reichenden Bedeutung vom IntVG als Vertragsänderung qualifiziert. Daher wird auf diese Regelung im Rahmen mit der Mitwirkung des Bundestages und Bundesrates im Vertragsänderungsverfahren näher einzugehen sein.

\section{Einbindung in die Subsidiaritătskontrolle}

Nach Art. 12 lit. b EUV sind die Parlamente der Mitgliedstaaten dazu aufgerufen, an der Wahrung des Gnundsatzes der Subsidiarität mitzuwirken. Auch hinsichtlich dieser Einbindung in die Subsidiaritätskontrolle ist zwischen den unionsrechtlichen Vorgaben auf der einen Seite und den nationalrechtlichen, in diesem Kontext also deutschen Vorgaben, auf der anderen Seite zu differenzieren. 


\section{Unionsrechtliche Vorgaben}

Der nun in Art. 5 Abs. 1 S. 2, Abs. 3 EUV enthaltene Subsidiaritätsgrundsatz gehört zu den wichtigsten Prinzipien des Unionsrechts. Soweit möglich, sollen Ziele auf lokaler, regionaler oder nationaler Ebene durch die Mitgliedstaaten verwirklicht werden. Nur wenn eine Verwirklichung auf Unionsebene wirkungsvoller ist, soll die Union von einer konkurrierenden Kompetenz Gebrauch machen können, wodurch regionale und nationale Identitäten bewahrt und eine Zentralisierung der Europäischen Union verhindert werden sollen. ${ }^{13}$

Nach Art. 1 des Subsidiaritätsprotokolls sind alle Unionsorgane zur Beachtung dieses Grundsatzes verpflichtet. Die nationalen Parlamente sollen zur Wahrung der Grundsätze aber in den Rechtsetzungsprozess einbezogen werden, liegt es doch gerade im Interesse der Mitgliedstaaten und ihrer Volksvertretungen, dass die Befugnisse dort und nicht auf Ebene der Europäischen Union ausgeübt werden. Aus diesem Grunde haben sie innerhalb eines Frühwarnsystems ex ante die Möglichkeit, mit der Subsidiaritätsrüge auf etwaige Verletzungen des Subsidiaritätsprinzips hinzuweisen und sie zu verhindern, sowie ex post die Möglichkeit, vermeintliche Verletzungen des Subsidiaritätsprinzips mit der Subsidiaritätsklage vor den EuGH zu bringen.

\section{a) Subsidiaritätsrüge innerhalb eines Frühwarnsystems}

Mit dem Frühwarnsystem hinsichtlich der Nichteinhaltung des Subsidiaritätsgrundsatzes durch geplante Rechtsakte der Europäischen Union wird den mitgliedstaatlichen Parlamenten die Möglichkeit gegeben, die Einhaltung oder Verletzung des Subsidiaritätsgrundsatzes vor Erlass des beabsichtigten Gesetzgebungsaktes zu überprüfen. Art. 4 des Subsidiaritätsprotokolls verpflichtet die Unionsorgane, ihre Entwürfe bzw. geänderten Entwürfe von Gesetzgebungsakten sowie legislative Entschließungen den nationalen Parlamenten zuzuleiten. Diese werden also umfassend über die Rechtsetzungsaktivitäten der Unionsorgane informiert. Über diese bloße Unterrichtungspflicht hinaus schreibt Art. 5 des Subsidiaritätsprotokolls den Unionsorganen ebenfalls vor, den nationalen Parlamenten auch Begründungen und Vermerke hinsichtlich der Grundsätze der Verhältnismäßigkeit und der Subsidiarität zugänglich zu machen, damit sie die Einhaltung dieser Grundsätze besser beurteilen können. Zugleich soll durch die Be- 
gründungspflicht auch eine gewisse Selbstïberprüfung der Unionsorgane erreicht werden. ${ }^{14}$

Die Parlamente der Mitgliedstaaten haben nach Art. 3 des Protokolls über die Rolle der nationalen Parlamente i.V.m. Art. 6 des Subsidiaritätsprotokolls anschließend acht Wochen Zeit, um die Entwürfe auf jhre Konformität mit dem Subsidiaritätsgrundsatz zu überprüfen und Verstöße in einer begründeten Stellungnahme darzulegen, die an den Präsidenten von Rat, Kommission und Europäischem Parlament zu richten ist. Dieses Vorgehen nach Art. 6 des Subsidiaritätsprotokolls wird auch als Subsidiaritätsrüge bezeichnet. Nach Art. 6 Abs. 1 S. 2 Subsidiaritätsprotokoll obliegt es den nationalen Parlamenten, auch die regionalen Parlamente mit Gesetzgebungskompetenzen, d.h., die deutschen Landtage, zur Frage der Subsidiarität zu konsultieren, womit erstmals im europäischen Primärrecht die regionalen Parlamente mit einbezogen werden. ${ }^{15}$ Eine genaue oder zwingende Mitwirkung wird den Regionalparlamenten bei der Subsidiaritätsrïge freilich hierdurch nicht zugedacht.

In Art. 7 des Subsidiaritätsprotokolls ist detailliert dargestellt; wie die Unionsorgane mit derartigen Stellungnahmen der nationalen Parlamente zu verfahren haben. Die Vorschrift trifft zahlreiche Differenzierungen in Abhängigkeit davon, wie viele nationale Parlamente eine begründete Stellungnahme zu einer Verletzung des Subsidiaritätsgrundsatzes eingereicht haben. Zur Gewichtung dieser Stellungnahmen erhält nach Art. 7 Abs. 1 UAbs. 2 jedes nationale Parlament zwei Stimmen, was vor allem dem Zweikammersystem in zahlreichen Mitgliedstaaten geschuldet ist, wo jeder Kammer eine Stimme zusteht. Zusammenfassend werden folgende Konstellationen unterschieden:

Erreicht die Anzahl der derart gewichteten Stimmen der nationalen Parlamente, die einen Verstoß gegen das Subsidiariatatsprinzip behaupten, kein im Protokoll festgelegtes Quorum, so haben die am Rechtsetzungsverfahren beteiligten Unionsorgane gemäß Art. 7 Abs. 1 des Subsidiaritätsprotokolls die Stellungnahmen lediglich zu berücksichtigen, wobei dies keine inhaltliche Auseinandersetzung mit der jeweiligen Stellungnahme verlangt. ${ }^{16}$

14 Wohland, Bundestag. Bundestat und Landesparlamente im europäischen Integrationsprozess, 2007, S. 206.

15 Wohland, Bundestag. Bundestal und Landesparlamente im europäischen Integrationsprozess, 2007, S. 199.

16 Kaufmann-Bühler, in: Lenz/Borchardt, EU-Verträge, Art. I2 ELV Rn. 11. 
Ist der Entwurf nach der Stellungnahme wenigstens eines Drittels der Stimmen der nationalen Parlamente nicht mit dem Subsidiaritätsgrundsatz in Einklang zu bringen, so verlangt Art. 7 Abs. 2 des Subsidiaritätsprotokolls von den Unionsorganen eine Überprüfung des Entwurfs. Dem Wortlaut nach ist hier also sehr wohl eine inhaltliche Auseinandersetzung mit den Stellungnahmen zu verlangen, die Unionsorgane müssen ihren Entwurf im Hinblick auf die Wahrung des Subsidiaritātsgrundsatzes überdenken. Das Quorum reduziert sich hierbei auf ein Viertel der Stimmen der nationalen Parlamente, wenn der beanstandete Entwurf auf Art. 61 lit. i AEUV beruht und Materien der Freiheit, der Sicherheit und des Rechts betrifft.

Erreichen die nationalen Parlamente, die in einer Stellungnahme von der Unvereinbarkeit von Entwurf und Subsidiaritätsgrundsatz ausgehen, die einfache Mehrheit der Stimmen, so muss der Entwurf ebenfalls von den Unionsorganen einer Prüfung unterzogen werden. Nach der Überprüfung ist es an der Kommission zu entscheiden, ob sie an diesem Vorschlag festhalten will. Hält die Kommission an dem Vorschlag fest, so muss sie darüber hinaus in einer begründeten Stellungnahme ihrerseits darlegen, warum der Entwurf ihrer Ansicht nach den Subsidiaritätsgrundsatz nicht verletzt. Diese Stellungnahme wird zusammen mit den Stellungnahmen der nationalen Parlamente dem Rat und dem Europäischen Parlament vorgelegt, damit diese alle Stellungnahmen im Rahmen des weiteren Rechtsetzungsverfahrens berücksichtigen können. Sind dann 55\% der Mitglieder des Rates oder 55\% der abstimmenden Mitglieder des Europäischen Parlaments der Ansicht, dass der Entwurf nicht mit dem Subsidiaritätsprinzip in Einklang steht, so wird der Gesetzgebungsvorschlag nicht weiter geprüft, das bedeutet, dass der Gesetzgebungsentwurf gescheitert ist.

Allerdings besteht natürlich auch die Möglichkeit, dass Rat und Europäisches Parlament über die Bedenken der nationalen Parlamente hinweg den Rechtsetzungsakt beschließen. An dieser Möglichkeit wird der konsulative Charakter der Subsidiaritätsrüge deutlich: Die nationalen Parlamente können lediglich ihre Bedenken geltend machen und versuchen, auf die Organe der unionsrechtlichen Rechtsetzung Einfluss zu nehmen - den Rechtsetzungsakt selbst aber können auch alle nationalen Parlamente gemeinsam und auch dann nicht verhindern, ${ }^{17}$ wenn sie der Ansicht sind, dass der Rechtsetzungsakt den Subsidiaritätsgrundsatz verletzt. 
b) Subsidiaritätsklage vor dem EuGH

In diesem Fall sind die nationalen Parlamente auf die Möglichkeit des Rechtsschutzes vor dem EuGH verwiesen. Gebrauch gemacht werden kann von diesem Rechtschutz erst nach Beschluss des umstrittenen Rechtssetzungsaktes, worauf schon der Wortlaut des Art. 8 Subsidiaritätsprotokoll hinweist. ${ }^{18}$ Nach Art. 8 des Subsidiaritätsprotokolls kann jedes nationale Parlament bzw. je nach innerstaatlicher Ausgestaltung jede Parlamentskammer vor dem EuGH wegen eines Verstoßes gegen das Subsidiaritätsprinzip klagen. Dieses Institut wird als Subsidiaritätsklage bezeichnet, die systematisch aber als normale Nichtigkeitsklage nach Art. 263 AEUV zu behandeln ist, wie Art. 8 des Subsidiaritätsprotokolls auch zum Ausdruck bringt. Formal wird die Subsidiaritätsklage deshalb als Klage des jeweiligen Mitgliedstaates und nicht etwa als Klage eines einzelnen Organs zu behandeln sein. ${ }^{19}$ Der Mitgliedstaat - vertreten durch seine Regierung - fungiert dabei allerdings lediglich als Bote und darf die Klage inhaltlich nicht verändern, was sich auch im Wortlaut ausdrückt, dass er die Klage ,im Namen des Parlaments übermittelt. ${ }^{\text {‘र2 }} \mathrm{Im}$ Übrigen ist darauf hinzuweisen, dass die Erhebung der Subsidiaritätsklage nicht von der vorherigen Erhebung einer Subsidiaritātsrüge abhängig ist. ${ }^{21}$

\section{Innerstaatliche Ausgestaltung in der Bundesrepublik Deutschland}

Innerstaatlich sind Subsidiaritätsrüge und Subsidiaritätsklage im Integrationsverantwortungsgesetz (IntVG) ausgestaltet. Allerdings dienen die schon angesprochene Unterrichtung nach $\$ \S 4$ und 5 EUZBBG, $\S 2$ EUZBLG sowie die damit einhergehende Einschätzung der Bundesregierung zur Einhaltung des Subsidiaritäts- und Verhältnismäßigkeitsprinzips nach $\S 7$ Abs. 1 S. 2, Abs. 2 EUZBBG der Vorbereitung einer Subsidiaritätsrüge.

18 Molsberger; Das Subsidiaritätsprinzip im Prozess der europäischen Konstitutionalisierung, 2009, S. 214.

19 Frenz, Jura 2010, 641, 644; Calliess, ZG 2010, 1, 11.

20 Molsberger. Das Subsidiaritätsprinzip im Prozess der europäischen Konstitutionalisierung, 2009, S. 216.

21 Wohland, Bundestag. Bundesrat und Landesparlamente im europäischen Integrationsprozess, 2007, S. 208; Callies, ZG 2010, 1, 12 mwN. 
a) Subsidiaritätsrüge

Hervorzuheben ist, dass die Subsidiaritätsrüge, wie $\S 11$ IntVG die begründete Stellungnahme i.S.d. Art. 6 Subsidiaritätsprotokolls bezeichnet, vom Bundestag wie vom Bundesrat jeweils selbständig erhoben werden kann, wobei der Gesetzgeber die genauere Verfahrensausgestaltung der Geschäftsführungsautonomie der beiden Organe überlassen hat. Nach $\$ 93$ c GOBT wird die Entscheidung über die Erhebung einer Subsidiaritätsrüge vom Bundestag getroffen, wobei sie auch auf den Ausschuss für die Angelegenheiten der Europäischen Union übertragen werden kann, den der Bundestag in Übereinstimmung mit $\S 2 \mathrm{~S}$. 1 EUZBBG errichtet hat. Mangels anders lautender Regelungen sind die Beschlüsse über die Erhebung der Subsidiarität mit der Mehrheit zu fassen. Auch das EUZBLG und die Geschäftsordnung des Bundesrates machen bezüglich einer Rügeerhebung durch den Bundesrat insofern keine genaueren Angaben.

\section{b) Subsidiaritătsklage}

Die Subsidiaritätsklage hat verfassungsrechtlich ihre Grundlage in Art. 23 Abs. la GG, der erst im Jahre 2010 als Reaktion auf das Urteil des Bundesverfassungsgerichts zum Vertrag von Lissabon eingefügt wurde. Auch hiernach sind Bundestag und Bundesrat unabhängig voneinander zur Klageerhebung berechtigt.

aa) Klageerhebung durch den Bundestag

Nach Art. 23 Abs. 1a S. 2 GG ist der Bundestag auf Antrag eines Viertels seiner Mitglieder sogar zur Klageerhebung verpflichtet.

Diese Regelung wirft Fragen hinsichtlich ihrer Verfassungsmäßigkeit wie auch ihrer Unionsrechtskonformität auf, weicht diese Regelung doch vom demokratischen Mehrheitsprinzip ab. ${ }^{22}$ Das Bundesverfassungsgericht ist in seinem Urteil zum Vertrag von Lissabon auf diese Problematik eingegangen. Es sieht Art. 23 Abs. la S. 2 GG - oder vielmehr die Verpflichtung, im Namen der Minderheit von wenigstens einem Viertel im Namen des Bundestages Subsidiaritätsklage zu erheben - als Mittel, der oppositionellen Parlamentsminderheit als Gegenspieler der Regierungsmehrheit den Rechtsweg zum Europäischen Gerichtshof zu eröff- 
nen. Die Abweichung vom Mehrheitsprinzip wird als verfassungsrechtlich unbedenklich angesehen, weil es nicht um eine Entscheidung mit regelnder Wirkung, sondern nur um die Anrufung eines Gerichts gehe. ${ }^{23}$ An dieser Stelle verweist der Zweite Senat auch auf Art. 93 Abs. 1 Nr. 2 GG, der die Anrufung des Bundesverfassungsgerichts ebenfalls auf Initiative eines Quorums von einem Viertel der Mitglieder des Bunđestages zulässt.

Zweifel werden in erster Linie hinsichtlich der Vereinbarkeit des Antragsrechts der Minderheit mit dem Unionsrecht geäußert. Zum einen ergebe sich das Problem, dass sich der Bundestag im Außenverhältnis den Willen einer Minderheit zurechnen lassen müsse, vor allem aber werde das Klagerecht, das unionsrechtlich nur dem Parlament zustehe. vom nationalen Recht unzulässigerweise erweitert. ${ }^{24}$ Es bedürfe auch keines besonderen Minderheitenschutzes, da sich die nationalen Parlamente hinsichtlich der Durchsetzung des Subsidiaritätsprinzips in einer natürlichen Kontroll- und Oppositionsrolle gegenüber der Unionslegislative befänden, ${ }^{25}$ ein möglicher Interessengegensatz zwischen Regierungsmehrheit und Opposition also zu verneinen sei. Letztlich wird auch ein Missbrauchsargument gegen das Recht der Parlamentsminderheit auf Klageerhebung geltend gemacht, da die Subsidiaritătsklage von einzelnen, europaskeptischen oder extremistischen Fraktionen nur zum Zwecke der Aufmerksamkeitserregung instrumentalisiert zu werden drohe. ${ }^{26}$

Dem wird entgegengesetzt, dass Rechtsschutz gerade auch Minderheitenschutz ist. ${ }^{27}$ Außerdem sei fraglich, ob die nationalen Parlamente dem Unionsgesetzgeber tatsächlich einheitlich in der erwähnten Oppositions- und Kontrollrolle gegenüberstehen oder ob nicht doch ein kollusives Zusammenwirken der Bundesregierung und der sie tragenden Parlamentsmehrheit zu erwarten ist. ${ }^{28}$ Weiterhin wird argumentiert, dass Art. 8 Abs. 1 Subsidiaritätsprotokoll auf die innerstaatliche Rechtsordnung verweist und damit die Entscheidung über die Ausgestaltung des parlamentarischen Klagerechts den Mitgliedstaaten selbst überlassen werde. ${ }^{29}$ Angesichts der nicht fern liegenden Erwägung, dass die parlamentarische Mehrheit aus Rücksicht auf die von ihr getragene Regierung und den von dieser Regierung entsandten Ratsvertreter auf eine Subsidiaritätsklage verzichte. könne

24 Uerpmann-Wittack/Edenharter, EuR 2009, 313, 319, 321.

25 Uerpmann-Wittzack/Edenharter, EuR 2009, 313, 325.

26. Uerpmann-Witzack/Edenharter, EuR 2009, 313, 327.

27 F.Kirchhof, DÖV 2004, 893, 895.

28 Gas, DÖV 2010, 313,317.

29 Frenz, Jura 2010, 641, 645. 
der innerstaatliche Ausgestaltungsspielraum nicht derart eingeschränkt werden. ${ }^{30}$ Schließlich habe die Parlamentsmehrheit bereits über die Möglichkeit der politischen Einflussnahme auf eben die von ihr getragene Regierung eine mittelbare Klagemöglichkeit zum EuGH. Im Ergebnis müsse auf jeden Fall verhindert werden, dass Minderheitenrechte ihrer Wirksamkeit durch einen Mehrheitsbeschluss beraubt werden."

Zum Teil wird vermittelnd argumentiert, dass zwar ein Viertel der gesetzlichen Mitglieder des Bundestages ausreichen sollten, es aber dennoch einer Mehrheitsentscheidung in der konkreten Abstimmung bedürfe. ${ }^{32}$

Für die Zulässigkeit der Klageerhebung aufgrund eines Minderheitenantrages spricht aber, dass Art. 8 des Subsidiaritätsprotokolls ausdrücklich auf die innerstaatliche Rechtsordnung eines jeden Mitgliedstaates verweist, diesen also grundsätzlich die Ausgestaltung der Klageerhebung im Namen ihrer Parlamente selbst überlässt. Gegen einen Verstoß gegen das unionsrechtliche Mehrheitsprinzip lässt sich auch hier anführen, dass es eben nicht um eine Entscheidung geht, die Auswirkungen auf das Rechtssetzungsverfahren selber hätte, sondern um die Wahrnehmung von Rechten, die auch einer Minderheit zustehen. Das Mehrheitsprinzip ist letztlich Teil des Demokratieprinzips, das eine Rückkoppelung von rechtlich verbindlichen Akten an einen Mehrheitswillen verlangt. ${ }^{33}$ Bei der Wahrnehmung von Rechten, die im Ergebnis lediglich auf ein im Einklang mit dem schon bestehenden Recht stehendes Urteil zielt, ist eine solche demokratische Rückkoppelung nicht erforderlich. Hier soll kein neues allgemeinverbindliches Recht geschaffen werden, sondern nur die Vereinbarkeit mit einem objektiven Rechtssatz überprüft werden. Ein bloßer Antrag im Rechtsschutzverfahren bedarf keiner Legitimation durch einen Mehrheitswillen. Letztlich wird das Klagerecht auch nicht ausgeweitet auf einzelne Abgeordnete oder Fraktionen, sondern es ist ein bestimmtes Quorum erforderlich, bei dessen Erreichen der deutsche Verfassungsgeber davon ausgeht - und im Rahmen seines innerstaatlichen Ausgestaltungsspielraumes nach Art. 8 Abs. 1 Subsidiaritätsprotokoll auch ausgehen darf, - dass gewichtige sachliche Bedenken hinsichtlich der Einhaltung des Subsidiaritätsprinzips bestehen, die nicht nur Ausdruck politischer Partikularinteressen sind. Es bleibt auch dabei, dass der Bundestag und nicht nur ein

31 Calliess, ZG 2010, 1, $30 \mathrm{f}$.

32 Kaufmann-Bühler, in: Lenz/Borchardt, EU-Verträge, Art. 12 EUV Rn. 13.

33 Baach, Parlamentarische Mitwirkung in Angelegenheiten der Europäischen Union, 2008, S. 46 . 
Teil des Bundestages Antragsteller ist. Somit ist von der verfassungs- wie unionsrechtlichen Zulässigkeit der Minderheitenregelung auszugehen.

Nicht ausdrücklich in $\$ 12$ Abs. 1 IntVG enthalten ist die vom Bundesverfassungsgericht aufgeworfene Problematik, dass die nationalen Parlamente die der Subsidiarität vorgelagerte Frage der Zuständigkeit der Europäischen Union überprüfen können sollten. ${ }^{34}$ Der Europäische Gerichtshof muss sich im Rahmen der Subsidiaritätsklage auf jeden Fall mit der Frage auseinandersetzen, ob eine aussch]jeßliche Zuständigkeit der EU besteht, weil sich in diesern Fall das rechtliche Problem der Subsidiarität nicht stellen würde..$^{35}$ Dies verlangt aber nicht zwingend eine komplette Zuständigkeitsprïfung.

Zum Teil wird unter Hinweis auf den Wortlaut des Art. 8 Subsidiaritätsprotokolls nur eine Rüge des Verstoßes gegen das Subsidiaritătsprinzip für zulässig erachtet. ${ }^{36}$ Für eine Geltendmachung von Kompetenzüberschreitungen der EU sei die ,allgemeine“ Nichtigkeitsklage der richtige Rechtsbehelf. ${ }^{37}$ Darüber hinaus seien Kompetenzfragen im Gegensatz zu Subsidiaritätsfragen juristisch hoch komplex und daher nicht geeignet, Gegenstand der Subsidiaritätskontrolle eines Parlaments zu sein. ${ }^{38}$

Für eine Einbeziehung der Kompetenz der EU in den Prüfungsumfang der Subsidiaritätsklage spricht aber, dass diese Klage auf eine wirksame Kontrolle und Begrenzung der Handlungen der Unionsorgane zugunsten der Mitgliedstaaten zielt und eine effektive Verfolgung dieser Ziele ohne eine Kompetenzkontrolle kaum möglich ist. ${ }^{59}$ Daneben stellt ein unionsrechtliches Handeln ohne Rechtsgrundlage oder Kompetenztitel zugleich auch einen Verstoß gegen das Subsidiaritätsprinzip dar, da in diesen Fällen ebenfalls die Regelungszuständigkeit der Mitgliedstaaten becinträchtigt wird. ${ }^{40}$ Dass Kompetenzfragen im Gegensatz zur Subsidiarität zu komplex seien, erscheint auch wenig überzeugend, da schließlich in beiden Fällen rechtliche Subsumtionen anzustellen sind. Und sofern

34 BVerfGE $123,267,383 \mathrm{f}$.

35 Wohland, Bundestag, Bundesrat und Landesparlamente im europäischen Integrationsprozess, 2007, S. 207.

36 Molsherger, Das Subsidiarilätsprinzip itn Prozess der europäischen Konstitutionalisierung, 2009, S. 224; Altmeier, in FS für Meyer, Derra (Htg.), 2006, S. 319.

37 Molsberger, Das Subsidiaritätsprinzip im Prozess der europäischen Konstitutionalisienung. 2009, S. 226.

38 Uerpmann-Wittack, EuGRZ 2009, 461, 463.

39 Frenz, Jura 2010. 64I, 644.

40 Shinani, JZ 2010, 753, 757. 
Kompetenzfragen als primär politisches Problem begriffen werden, ist das Parlament in besonderem Maße das geeignete Organ zu ihrer Klärung.

bb) Klageerhebung durch den Bundesrat

Dic Klageerhebung durch den Bundesrat hingegen ist durch Art. 23 Abs. la S. 2 GG nicht näher ausgestaltet. Nach § 12 Abs. 2 IntVG ist das Verfahren über die Klageerhebung der Geschäftsordnung des Bundesrates vorbehalten. Mangels abweichender Regelungen gilt bei der Beschlussfassung über die Erhebung der Subsidiaritätsklage das Mehrheitsprinzip, wobei es eine informelle Absprache der Ministerpräsidenten gibt, das Klagebegehren eines Landes regelmäßig zu unterstützen. ${ }^{4 !}$ Zwar wird eine Ausdehnung des Antragsrechts auf eine Minderheit auch für den Bundesrat diskutiert, jedoch fast einhellig abgelehnt:

Zunächst ist das Mehrheitsprinzip für den Bundesrat in Art. 52 Abs. 3 S. 1 GG verankert. Zwar könnte eine Abweichung von diesem Prinzip nach Art. 23 Abs. la S. $3 \mathrm{GG}$ vorgenommen werden. In $\S 12 \mathrm{Abs} .2$ IntVG ist dies aber nicht geschehen. Hätte der Gesetzgeber die Befugnis zur Abweichung vom Mehrheitsprinzip auf den Geschäftsordnungsgeber delegieren wollen, so hätte er dies deutlich machen müssen, was er jedoch in $\S 12$ Abs. 2 IntVG nicht getan hat und auch nicht tun wollte. ${ }^{42}$ Hinzu kommt, dass die Erwägungen, die das Klagerecht einer Minderheit des Bundestages stützen, nicht auf den Bundestag übertragbar sind. Da die Bundesregierung sich nicht auf eine Mehrheit im Bundesrat stützen muss und auch in der Staatspraxis eine Identität von Bundestags- und Bundesratsmehrheit zunehmend seltener wird, ist ein kollusives Zusammenwirken von Bundesratsmehrheit und Bundesregierung oder eine ungebotene Rücksichtnahme der Bundesratsmehrheit auf die Bundesregierung nicht in dem Maße zu befürchten wie beim Bundestag. ${ }^{43}$ Des Weiteren würde bei der Zusammensetzung des Bundesrates die unionsrechtlich vorgegebene Klagebefugnis unzulässigerweise auf eines oder eine Gruppe von Bundesländern erweitert. ${ }^{44}$

41 Kaufmann-Bühler, in: Lenz/Borchardt, EU-Verträge. Art. 12 EUV Rn. 13 mwN.

42 Uerpmann-Wittzack, EuGRZ 2009, 461, 467.

43 Gas, DÖV 2010, 313, 318.

44 Gas, DÖV 2010,313, 318. 
c) Sonstige Interventionsmöglichkeiten von Bundestag und Bundesrat

Über Art. 12 IntVG hinaus gibt es noch weitere Wege für Bundestag und Bundesrat, im europäischen Rechtssetzungsverfahren zu intervenieren.

aa) Notbremsenmechanismus

So kennt $\$ 9$ Abs. 1 IntVG den so genannten Notbremsenmechanismus, nach dem der deutsche Vertreter im Europäischen Rat diesen befassen muss, wenn ein Bundestagsbeschluss dies verlangt. $\S 9$ Abs. 2 IntVG lässt selbiges für einen Bundesratsbeschluss gelten, solange nicht die Materie der ausschließlichen Bundeskompetenz unterfiele und auch keine Zustimmung des Bundesrates erforderlich wäre. Der Notbremsenmechanismus gilt jedoch nur in bestimmten Bereichen, nämlich beim Sozialversicherungsrecht im Rahmen der Arbeitnehmerfreizügigkeit, des Strafverfahrensrechts und der Schwerkriminalität. Zurückzuführen ist diese Regelung auf den Notbremsenmechanismus des AEUV, demzufolge die Mitglieder des Rats Rechtssetzungsentwürfe dem Rat vorlegen dürfen, wenn sie der Ansicht sind, dass die als sensibel und genuin hoheitlich empfundenen Grundsätze der sozialen Sicherungssysteme und der Strafrechtsordnung betroffen sind.

Durch die Ratsvorlage wird das Rechtssetzungsverfahren ausgesetzt. Aus $§ 9$ IntVG folgt, dass die Bundesregierung in Gestalt des Vertreters im Rat an Weisungen der gesetzgebenden Organe zur Auslösung des Notbremsenmechanismus gebunden ist. Da $\$ 9$ IntVG keine Gesetzesform vorschreibt, kann die Weisung in Form eines einfachen Beschlusses erfolgen. ${ }^{45}$ Andererseits verbleibt auch der Bundesregierung die Möglichkeit, ohne Weisung von Bundestag oder Bundesrat den Notbremsenmechanismus auszulösen, wozu diese beiden Organe nach Art. 23 Abs. 3 oder Abs. 5 GG Stellungnahmen abgeben können. ${ }^{46}$

bb) Nichtigkeitsklage bei ausschließlichen Länderkompetenzen

Nach $\S 7$ Abs. 1 EUZBLG erhebt die Bundesregierung auf Verlangen des Bundesrates Klage zum Europäischen Gerichtshof, sofern ausschließliche Gesetzgebungskompetenzen der Länder berührt sind. Auch diese Einbindung des Bundesrates in das Rechtsschutzsystem vor dem Europäischen Gerichtshof ist unions-

46 Nellesheim, NJW 2010, 177, 181. 
rechtlich nicht vorgeschrieben. Umfasst sind hiervon nicht nur die Subsidiaritätsklage, die der Bundesrat schließlich selbständig erheben kann, sondern alle Klagemöglichkeiten, die zur Geltendmachung einer Verletzung von ausschließlichen Gesetzgebungsbefugnissen der Länder erhoben werden können. Die Norm stellt daher weniger eine Stärkung des parlamentarischen Organs Bundesrat dar, sondern sichert vielmehr die Wahmehmung von Interessen der Länder durch die klageberechtigte Bundesrepublik.

III. Einbindung der nationalen Parlamente in den Raum der Freiheit, der Sicherheit und des Rechts

Neben den allgemeinen Informationsrechten und ihrer Einbindung in die Subsidiaritätskontrolle wirken die nationalen Parlamente gemäß Art. 12 lit. c EUV auch im Rahmen des Raums der Freiheit, der Sicherheit und des Rechts mit. Hier werden die Parlamente insbesondere in die politische Kontrolle von Europol und die Bewertung der Tätigkeit von Eurojust, also der Zusammenarbeit der Staatsanwaltschaften, einbezogen. Im Gegensatz zu Art. 12 lit. b EUV handelt es sich hierbei aber nicht um eine Mitwirkung im Bereich der Rechtsetzung, sondern um eine Beteiligung an Mechanismen zur Bewertung der Durchführung von Unionspolitiken. Eine für eine demokratische Legitimation hinreichende Kontrolle durch die Parlamente und eine ausreichende Beachtung des Subsidiaritätsprinzips sollen und können hierdurch nicht gewährleistet werden. ${ }^{47}$

Freilich sind auch bezüglich von Maßnahmen im Bereich des Raums der Freiheit, der Sicherheit und des Rechts die allgemeinen Informations- und Berichtspflichten zu beachten, soweit der Anwendungsbereich von EUZBBG und EUZBLG eröffnet ist.

IV. Rolle der mitgliedstaatlichen Parlamente im Bereich der Vertragsänderung

Besondere Legitimationsaspekte hat es dagegen, wenn die nationalen Parlamente nach Art. 12 lit. d EUV an den Vertragsänderungsverfahren nach Art. 48 EUV zu beteiligen sind.

47 Calliess, ZG 2010, 1, 15; Kretschmer, in: Vedder/Heintschel v. Heinegg. EVV, Art. III273 Rn. 10. 


\section{Ordentliches Vertragsänderungsverfahren}

Die der EU zugrunde liegenden primärmechtlichen Verträge, EUV und AEUV, stellen der Sache nach völkerrechtliche Vereinbarungen zwischen den Mitgliedstaaten dar. Änderungen der Verträge sind von den Mitgliedstaaten gemäß ihren jeweiligen verfassungsrechtlichen Vorgaben zu ratifizieren. In der Bundesrepublik Deutschland müssen die Gesetzgebungsorgane hierzu ein Gesetz erlassen, das nicht nur den formellen Anforderungen des Art. 59 Abs. 2 GG, sondern vor allem auch den materiellen Anforderungen des Art. 23 Abs. 1 S. 2 GG entspricht, da Vertragsänderungsverfahren wie eine Übertragung von Hoheitsrechten zu behandeln sind. ${ }^{48}$ Regelmäßig werden bei Vertragsändenungsverfahren sogar Gesetze mit verfassungsändemden Mehrheiten gemäß Art. 23 Abs. 1 S. 3 GG erforderlich sein, da eine Änderung der primärrechtlichen Grundlagen Zuständigkeitsgrenzen verschiebt und damit materiell verfassungsändernd wirkt. ${ }^{49}$

Dieses grundgesetzlich vorgesehene Verfahren der Vertragsändenung ist in Art. 48 Abs. 1-4 EUV als ordentliches Vertragsänderungsverfahren festgelegt. Die nationalen Parlamente werden gemäß Art. 48 Abs. 4 EUV im Rahmen der verfassungsrechtlichen Vorschriften des jeweiligen Mitgliedstates bei der Ratifikation gemäß Art. 48 Abs. 4 EUV relevant. Im Änderungsverfahren selbst können die nationalen Parlamente aber auch schon im Rahmen der so genannten Konventslösung nach Art. 48 Abs. 3 EUV eine Rolle spielen. Der Rat kann einen Konvent einberufen, wenn Europäisches Parlament oder Kommission Änderungsvorschläge gemacht haben. Dieser Konvent, dem unter anderem auch Vertreter der nationalen Parlamente angehören, erarbeitet im Konsensverfahren eine Empfehlung, die wiederum einer Regierungsverireterkonferenz vorgelegt wird, die die Vertragsänderungen dann vereinbart. Auf diese Weise können die nationalen Parlamente Einfluss auf den Inhalt von Vertragsänderungen nehmen, wobei dieser in Anbetracht des beschriebenen Verfahrens sehr mittelbar bleibt. Die Letztentscheidenden über Vertragsänderungen bleiben die Mitgliedstaaten in Form der Regierungsvertreterkonferenz.

\section{Vereinfachte Vertragsänderungsverfahren}

Neben diesem ordentlichen Vertragsänderungsverfahren sehen Art. 48 Abs. 6 und 7 EUV das so genannte vereinfachte Änderungsverfahren vor.

49 Geiger, inl: Geiger/Khan/Kolzur, EUV/AEUV, Art. 48 EUV Rn. 11. 
Nach Art. 48 Abs. 6 EUV kann der Europäische Rat in Abweichung vom ordentlichen Vertragsänderungsverfahren nach Anhörung des Europäischen Parlaments und der Kommission sogleich Änderungen des Dritten Teils des AEUV, der sich mit internen Politikbereichen der Union befasst, beschließen, wobei dieser Beschluss nicht zu einer Ausdehnung der Kompetenzen der EU führen darf. Der Beschluss tritt aber wiederum erst nach der Zustimmung durch die Mitgliedstaaten gemäß ihren verfassungsrechtlichen Bestimmungen in Kraft, so dass sich in Bezug auf die Mitwirkung der nationalen Parlamente keine Abweichungen vom ordentlichen Änderungsverfahren ergeben.

\section{a) Unionsrechtliche Vorgaben an Brückenklauseln}

Anders ist dies im Rahmen des vereinfachten Änderungsverfahrens nach Art. 48 Abs. 7 EUV bei Verwendung von so genannten Brückenklauseln oder Passerelle-Klauseln. Das bedeutet, dass durch das Änderungsverfahren nach Art. 48 Abs. 7 EUV für Beschlüsse des Rates, die bisher Einstimmigkeit verlangen, eine qualifizierte Mehrheit vorgesehen werden kann. Ebenso kann der Übergang vom besonderen zum ordentlichen Gesetzgebungsverfahren beschlossen werden. Die Vereinfachung gegenüber dem ordentlichen Vertragsänderungsverfahren besteht bei diesen Brückenklauseln darin, dass kein Ratifizierungsverfahren nach Maßgabe der verfassungsrechtlichen Bestimmungen der Mitgliedstaaten vorgesehen ist. Allerdings sind nach Art. 12 lit. d i.V.m. Art. 48 Abs. 7 UA 3 EUV die nationalen Parlamente mit in das Verfahren einzubeziehen.

Nach Art. 6 des Protokolls über die Rolle der nationalen Parlamente in der EU unterrichtet der europäische Rat die nationalen Parlamente mindestens sechs Monate, bevor er von Art. 48 Abs. 7 EUV Gebrauch macht. Der Europäische Rat. der das zuständige Initiativorgan für Verfahren nach Art. 48 Abs. 7 EUV ist, leitet den Entwurf den nationalen Parlamenten zu. Lehnt auch nur ein nationales Parlament den Vorschlag innerhalb von sechs Monaten ab, so kann der Europäische Rat den Beschluss nicht erlassen. Jedem einzelnen Parlament der Mitgliedstaaten kommt hier insofern ein Ablehnungs- und Vetorecht zu. Hierdurch wird der Verlust mitgliedstaatlicher Einflüsse infolge des fehlenden Ratifizierungserfordernisses kompensiert. ${ }^{50}$

Neben der allgemeinen Brückenklausel kennen EUV und AEUV noch spezielle Regelungen, die einen Übergang vom Einstimmigkeitserfordernis zur qualifizier- 
ten Mehtheit im Rat vorsehen, wic z.B. Art. 31 Abs. 3 EUV im Verfahren der GASP oder Art. 81 Abs. 3 AEUV im Bereich der justiziellen Zusammenarbeit in Familiensachen. Letztgenannte Vorschrift sieht ähnlich wie Art. 48 Abs. 7 EUV ein Ablehnungsrecht jedes nationalen Parlaments innerhalb von sechs Monaten nach Übermittlung des Vorschlages vor. Andere spezielle Brückenklauseln, wie z.B. der genannte Art. 31 Abs. 3 EUV oder Art. 153 Abs. 2 oder auch 312 Abs. 2 S. 2 AEUV sehen eine Einbeziehung der nationalen Parlamente nicht vor und werden auch nicht vom Verweis des Art. 12 lit. d EUV gedeckt.

b) Nationalrechtliche Anforderungen an Brückenklauseln in Deutschland

Für Deutschland setzt sich namentlich das IntVG mit den Brückenklauseln und den Verfahren zur Änderung der Verträge auseinander, nachdem das Bundesverfassungsgericht hier einen besonderen Handlungsbedarf zur Sicherstellung der demokratischen Legitimation gesehen hat. Es verlangt im Ergebnis eine sehr viel stärkere parlamentarische Einbindung als das Unionsrecht.

\section{aa) Vereinfachtes Änderungsverfahren nach Art. 48 Abs. 6 EUV}

Bemerkenswert ist, dass § 2 IntVG schon hinsichtlich des vereinfachten Änderungsverfahrens nach Art. 48 Abs. 6 EUV ein Gesetz nach Art. 23 Abs. 1 GG als notwendig für eine wirksame deutsche Zustimmung ansieht. Ob und wann eine verfassungsändernde Mehrheit nach Art. 23 Abs. 1 S. 3 GG erforderlich ist, führt $\S 2$ IntVG allerdings nicht näher aus, sondern überlässt dies der Einzelfallprüfung. Im Regelfall dürfte die Notwendigkeit eines Gesetzes nach Art. 23 Abs. 1 S. 3 GG wegen der materiellen Bedeutung dieser semi-autonomen Vertragsänderungen zu bejahen sein. ${ }^{51}$ Da das Zustimmungsgesetz zu einer Vertragsänderung nach Art. 48 Abs. 6 EUV als formelles Gesetz sowieso nur am Grundgesetz zu messen ist, kommt der Norm aber nur deklaratorische Bedeutung zu. ${ }^{52}$

bb) Weitere besondere Brückenklauseln

Neben dem Art. 48 Abs. 6 EUV kennt das europäische Primärrecht noch einige andere Vorschriften, die dieser nachgebildet sind, wie z.B. die Regelung über

52 Daiber, DÖV 2010, 293, 294. 
den Beitritt der EU zur EMRK in Art. 218 Abs. 8 UAbs. 2 S. 2 AEUV. Auch hier schreibt $\S 3$ Abs. 1 IntVG, ähnlich wie $§ 2$ IntVG, vor, dass die Zustimmung der Bundesrepublik durch ein Gesetz nach Art. 23 Abs. 1 GG zu erfolgen hat. $\S 3$ Abs. 2 IntVG trifft die gleiche Regelung für dort genannte Bestimmungen des Rates und betrifft Akte, die nicht in der Form eines Beschlusses nach Art. 288 AEUV erlassen werden. ${ }^{53}$

Eine besondere Regelung trifft $\S 3$ Abs. 3 IntVG hinsichtlich des Übergangs zur gemeinsamen Verteidigung der Mitgliedstaaten in Art. 42 Abs. 2 UA 1 EUV. Diese Norm sieht sowohl einen Beschluss des Europäischen Rates auch als eine Zustimmung der Mitgliedstaaten zur gemeinsamen Verteidigung vor, lässt aber nicht erkennen, ob der Beschluss des Europäischen Rates erst nach der Zustimmung der Mitgliedstaaten in Kraft tritt. ${ }^{54}$ Der deutsche Gesetzgeber hat auf diese Unsicherheit durch die Ausgestaltung einer doppelten parlamentarischen Mitwirkung reagiert. Nach $\S 3$ Abs. 3 S. 4 IntVG bedarf die Zustimmung der Bundesrepublik Deutschland eines Gesetzes nach Art. 23 Abs. 1 GG, worin die Regelung $\S 3$ Abs. 1 IntVG ähnelt. Zum anderen aber ist der deutsche Vertreter im Europäischen Rat nach $\S 3$ Abs. 1 S. 1 und 3 IntVG gehalten, seinc Zustimmung oder Enthaltung nur nach einem entsprechenden Bundestagsbeschluss zu erteilen. Ohne Bundestagsbeschluss muss er den Vorschlag im Europäischen Rat ablehnen. Damit kann der Bundestag den Beschluss einer gemeinsamen Verteidigung gegen den Willen der Bundesregierung verhindern.

$\S 4$ Abs. 1 IntVG regelt schließlich das innerstaatliche Verfahren hinsichtlich der Brückenklausel des Art. 48 Abs. 7 EUV. Da ein Übergang vom Einstimmigkeitserfordernis zur qualifizierten Mehrheit sowie vom besonderen zum ordentlichen Gesetzgebungsverfahren nur durch einen einstimmigen Beschluss des Europäischen Rates möglich ist, darf der deutsche Vertreter in diesem Gremium nur zustimmen oder sich enthalten, wenn ein Gesetz nach Art. 23 Abs. 1 GG vorliegt. Die Stimmenthaltung bedarf deswegen auch einer gesetzlichen Ermächtigung, weil eine solche nach Art. 235 Abs. 1 UAbs. 3 AEUV dem Zustandekommen eines einstimmigen Europäischen Ratsbeschlusses nicht entgegensteht. Im Gegensatz zum vorherigen Begleitgesetz wird ein Schweigen der gesetzgebenden Organe nicht mehr als Zustimmung gewertet. ${ }^{55}$ Ob das Gesetz nach Art. 23 Abs. 1 S. 3 GG einer verfassungsändernden Mehrheit bedarf, wird im IntVG hier

55 Calliess, ZG 2010, 1, 28. 
auch nicht vertieft, dürfte aber angesichts der in der Folge schwindenden Einflussmöglichkeit des Ratsvertreters zu bejahen sein. ${ }^{56}$

§ 4 Abs. 2 IntVG trifft für die Brückenklausel für die justizielle Zusammenarbeit im Familienrecht in Art. 81 Abs. 3 AEUV eine entsprechende Regelung. Zweifel hinsichtlich dieser Norm werden insofern angemeldet, als man aus Art. 81 Abs. 3 AEUV eine Ausweitung der Kompetenz der EU lesen könnte, was der Gesetzgeber offensichtlich nicht getan hat. Sollte man doch eine Kompetenzerweiterung annehmen, wäre hierfür insofern auch ein Gesetz nach Art. 23 Abs. 1 GG notwendig. ${ }^{57}$

Neben der Ermächtigung des deutschen Vertreters im Europäischen Rat hat das IntVG in $\$ 10$ auch das parlamentarische Ablehnungsrecht des Art. 48 Abs. 7 EUV näher ausgestaltet. ${ }^{5 \mathrm{~K}}$ Nach $\S 10$ Abs. I Nr. 1 IntVG kann der Bundestag die Ablehnung beschließen, wenn die Initiative des Europäischen Rates im Schwerpunkt ausschließliche Gesetzgebungszuständigkeiten des Bundes betrifft. In allen anderen Fällen können nach \$10 Abs. 1 Nr. 2 IntVG Bundestag oder Bundesrat die Ablehnung beschließen. Bundestag oder Bundesrat können in diesen Fällen unabhängig voneinander eine Ablehnung aussprechen. Somit genügt die Ablehnung nur eines der beiden Organe, um die Initiative des Europäischen Rates zu stoppen. Da das IntVG hier keine Gesetzesform fordert, kann die Ablehnung in Einklang einfach in Beschlussform ergehen.

Angesichts der zweispurigen Einwirkungsmöglichkeit von Bundesrat und Bundestag stellt sich die Frage, ob eine Ablehnung nach § 10 IntVG noch erklärt werden kann, nachdem der deutsche Ratsvertreter nach $\$ 4$ IntVG zu einer $\mathrm{Zu}$ stimmung ermächtigt wurde. Einerseits steht $\$ 10$ der Systematik nach hinter $\$ 4$ und die Ablehnung durch das. Parlament ist im Verfahren des Art. 48 Abs. 7 EUV unabhängig von der Stimmabgabe im Europäischen Rat. Auf der anderen Seite sieht das Verfahren nach Art. 48 Abs. 7 AEUV aber zuerst eine Befassung der nationalen Parlamente und erst abschließend eine Abstimmung im Europäischen Rat vor, so dass Bundestag und Bundesrat sich erst mit der Zustimmungsermächtigung befassen, nachdem sie über die Ablehnung zu entscheiden hatten. ${ }^{59}$ Des Weiteren spricht gegen eine Ablehnung nach einer erteilten Zustimmungsermächtigung, dass erstere durch einen einfachen Beschluss erfolgt und

56 Netlesheim, NJW 2010, 177, 179.

57 Daiber, DÖV 2010, 293, 297.

58 § 10 Abs. 3 IntVG erklär für die Brückenklausel des Art. 81 Abs. 3 AEUV § 10 Abs. 1 IntVG für entsprechend anwendbar.

59 Nettesheim, NJW 2010, 177, 179. 
aus rechtsstaatlicher Sicht nicht die in einem Gesetz nach Art. 23 Abs. 1 GG erklärte Zustimmung faktisch aufheben kann. ${ }^{60}$ Somit ist die Ansicht vorzugswürdig, dass nach einer erteilten Zustimmungsermächtigung keine Ablehnung nach $\S 10$ IntVG mehr erfolgen darf.

cc) National angeordnete parlamentarische Mitwirkungspflichten

Neben diesen Brückenklauseln gibt es in ELV und AEUV besondere Brückenklauseln, die keine Mitwirkung der nationalen Parlamente vorsehen. Das Bundesverfassungsgericht hält aber auch in diesen Fällen eine Mitwirkung von Bundesrat und Bundestag für geboten, wenn auch nicht unbedingt in der Form des Art. 23 Abs. 1 GG. ${ }^{61}$ Hierauf hat der Gesetzgeber durch die $\$ \S 5$ und 6 IntVG reagiert.

Nach $\S 5$ Abs. 1 und 6 Abs. 1 IntVG darf der deutsche Vertreter im (Europäischen) Rat bei den jeweils bezeichneten Brückenklauseln nur dann seine $\mathrm{Zu}$ stimmung erklären oder sich der Stimme enthalten, wenn der Bundestag hierzu einen Beschluss gefasst hat. Eines Gesetzes nach Art. 23 Abs. 1 GG bedarf es hier also nicht. Nach $\S 5$ Abs. 2 und $\S 6$ Abs. 2 i.V.m. $\S 5$ Abs. 2 IntVG bedarf es zusätzlich eines Beschlusses des Bundesrates, wenn nicht lediglich eine ausschließliche Gesetzgebungskompetenz des Bundes betroffen ist oder die Materie eine Zustimmmungsbedürftigkeit des Bundesrates auslösen würdc. Nicht erforderlich ist hierbei, dass die Mitwirkungstatbestände im Schwerpunkt betroffen sind, vielmehr genügt ein Randbezug zu Materien, die der Gesetzgebungskompetenz der Länder oder der Zustimmungsbedürftigkeit des Bundesrates unterfallen. ${ }^{62}$

Da die Bundesregierung im Gegensatz zum Gesetzgebungsverfahren nach Art. 23 Abs. 1 GG im Beschlussverfahren kein Initiativrecht besitzt, wird ihr dieses durch $\S 5$ Abs. 1 S. 2, 6 Abs. 1 S. 2 IntVG eingeräumt. Somit kann sie auch einen Zustimmungsbeschluss initiieren.

Im Kontext der vereinfachten Vertragsänderung scheint die Frage erörterungswert, auf welche Weise Bundestag und Bundesrat die im IntVG eingeräumten Befugnisse gerichtlich geltend machen können. Soweit sich die Organe im Or-

61 BVerfGE 123, 267, 392.

62 Hahn. EuZW 2009, 758, 761. 
ganstreitverfahren gemäß \$ 64 Abs. 1 BVerfGG darauf berufen können, in Rechten aus Art. 23 Abs. 1 GG verletzt zu sein, ist dies unproblematisch.

Schwierigkeiten ergeben sich dort, wo der Gesetzgeber des IntVG - in Nachzeichnung der Auslegung des Art. 23 Abs. 1 GG durch das Bundesverfassungsgericht - auf das Erfordemis eines solchen Gesetzes verzichtet und das Handeln des Regierungsvertreters von einem einfachen Beschluss abhängig gemacht hat wie in $\$ 5$ Abs. 2 IntVG. Aus der Lissabon-Entscheidung lässt sich insofern kein geltend zu machendes Recht von Bundestag und Bundesrat entnehmen, ${ }^{63}$ so dass es momentan keine befriedigende Lösung dieses Problems zu geben scheint. Eine Verletzung der Unterrichtungsrechte nach dem EUZBBG bzw. EUZBLG hingegen stellt eine Verletzung von Art. 23 Abs. 2 S. 2 GG dar und kann damit unproblematisch geltend gemacht werden.

\section{c) Mitwirkung der deutschen Gesetzgebungsorgane bei anderen Klauseln}

Das IntVG kennt neben den Brückenklauseln noch andere Klauseln der primärrechtlichen Verträge, bei denen zwar nicht das Unionsrecht, aber das Bundesverfassungsgericht eine parlamentarische Mitwirkung als zwingend ansieht. ${ }^{64}$ Die Mitwirkung hinsichtlich dieser Klauseln ist im IntVG geregelt.

Nach $\$ 7$ Abs. 1 IntVG darf der deutsche Vertreter im Rat einem Beschlussvorschlag hinsichtlich der Kompetenzerweiterungsklauseln auf dem Gebiet des materiellen Strafrechts und der Europäischen Staatsanwaltschaft (Art. 83 Abs. 1 UAbs. 3, 86 Abs. 4 AEUV) nur dann zustimmen oder sich enthalten, wenn hierzu ein Gesetz nach Art. 23 Abs. 1 GG in Kraft getreten ist. $\$ 7$ Abs. 2 IntVG wejtet dieses Erfordernis auch auf Satzungsänderungen der Europäischen Investitionsbank aus. Das Erfordernis eines Gesetzes nach Art. 23 Abs. 1 GG ist gerade auf dem Gebiet des Strafrechts und der Staatsanwaltschaft stimmig, weil die Kompetenzen der EL durch die bezeichneten Klauseln ausgewejtet werden können. ${ }^{65}$ Kritisch wird die fehlende Erfassung von Art. 82 Abs. 2 UAbs 2 lit. d) AEUV gesehen. ${ }^{66}$ Von der Regelungstechnik ist die Vorschrift stark an den $\$ 4$ Abs. 1 lntVG angelehnt.

64 BVerfGE 123, 267, 436.

65 Daiber, DÖV 2010, 293, 297.

66 Nettesheim, NJW 2010, 177, 180; Hahn, EuZW 2009, 758, 761. 
Eine parlamentarische Mitwirkung ist nun auch bezüglich der Flexibilitätsklausel des Art. 352 AEUV geregelt. Nach dieser Bestimmung kann der Rat der EU im Wege des einstimmigen Beschlusses Regelungen über die bestehenden Kompetenzgrenzen der EU hinaus treffen, soweit dies zur Verwirklichung der primärrechtlichen Vertragsziele notwendig ist. Hier drängt sich die Erforderlichkeit eines Zustimmungsgesetzes nach Art. 23 Abs. 1 GG schon wegen des Verbots der Übertragung der Kompetenz-Kompetenz auf die EU auf. ${ }^{67}$ Der Gesetzgeber hat in $\S 8$ IntVG die Zustimmung oder Enthaltung des deutschen Ratsvertreters konsequent von einem Gesetz nach Art. 23 Abs. 1 GG abhängig gemacht. Ob eine verfassungsändernde Mehrheit nach Art. 23 Abs. 1 S. 3 GG erforderlich ist, ist hier nicht näher geregelt und der Einzelfallbewertung überlassen. ${ }^{6 B}$

V. Beteiligung der mitgliedstaatlichen Parlamente an der Unionserweiterung

Als fünfte Form der Einbindung der nationalen Parlamente in Entscheidungen der Europäischen Union normiert Art. 12 lit. e EUV die Pflicht, die nationalen Parlamente über Anträge auf den Beitritt zur EU nach Art. 49 EUV zu unterrichten. Auch Art. 49 Abs. 1 S. 2 EUV schreibt diese Pflicht zur Unterrichtung der nationalen Parlamente über Beitrittsanträge fest. Darüber hinaus erwähnt das europäische Primärrecht eine Beteiligung der nationalen Parlamente an der Erweiterung der EU nicht ausdrücklich. Art. 49 EUV stellt jedoch klar, dass ein Beitritt eine Anpassung, d. h. Änderung der primärrechtlichen Verträge darstellt, die der Ratifikation der Mitgliedstaaten gemäß ihrer verfassungsrechtlichen Vorschriften bedarf. Somit ist für die Neuaufnahme eines Mitgliedes, die sich mangels anderer Regelungen im ordentlichen Änderungsverfahren vollzieht, nach deutschem Verfassungsrecht ein Gesetz nach Art. 23 Abs. 1 GG i.V.m. Art. 59 Abs. 2 GG erforderlich.

Hinsichtlich der Unterrichtung über einen Beitrittsantrag verweist $\S 10$ Abs. 1 EUZBBG auf den allgemein für alle Vorhaben geltenden $\$ 9$ EUZBBG und gewährt dem Bundestag somit zunächst ein Recht auf Stellungnahme. Nach $\S 10$ Abs. 2 S. 1 EUZBBG soll die Bundesregierung vor einem Beschluss im Rat Einvernehmen mit dem Bundestag herstellen, kann nach § 10 Abs. 2 S. 2 EUZBBG aber aus wichtigen außen- oder integrationspolitischen Gründen von der Entscheidung des Bundestages abweichen. Diese Vorschrift ähnelt dem bereits erwähnten $\S 9$ Abs. 4 S. 6 EUZBBG. Im Beratungsstadium hat der Bundestag so-

68 Nettesheim, NJW 2010, 177, 181. 
mit Einfluss-, aber noch keine Verhinderungsmöglichkeiten. Diese kommen ihm jedoch im abschließenden Gesetzgebungsverfahren nach Art. 23 Abs. 1 GG zu.

Der Bundesrat wird im Beratungsstadium nach Anlage Teil VII 2 unterrichtet, sobald der Rat beabsichtigt, einen Beschluss über Aufnahmeverhandlungen zu treffen und soweit Länderinteressen betroffen sein könnten. Der Anhang verweist auf $\$ 5$ EUZBLG, wonach der Bundesrat eine Stellungnahme abgibt, die der Vertreter der Bundesregierung im Rat berücksichtigt, soweit Interessen der Länder bei ausschließlicher Bundesgesetzgebungskompetenz berührt sind bzw. konkurrierende Gesetzgebung vorliegt (Abs. 1) und maßgeblich berücksichtigt, wenn im Schwerpunkt ausschließliche Gesetzgebungskompetenzen der Länder betroffen sind oder Länderbehörden oder -verwaltungsverfahren betroffen sind (Abs. 2). Im Ratifikationsverfahren ist der Bundesrat nach der alligemeinen Maßgabe des Art. 23 Abs. 1 S. 2 GG zu beteiligen.

\section{Interparlamentarische Zusammenarbeit}

Art. 12 lit. f EUV sieht schließlich vor, dass die nationalen Parlamente aktiv an der interparlamentarischen Zusammenarbeit untereinander sowie mit dem Europäischen Parlament mitwirken. Diese interparlamentarische Zusammenarbeit lässt sich als Element einer interparlamentarischen Demokratie im engeren Sinne begreifen. Sie ist insbesondere im Protokoll über die Rolle der nationalen Parlamente in der Europäischen Union năher ausgestaltet und vollzieht sich in erster Linie im Rahmen der Konferenz der Europa-Ausschïsse der Parlamente nach Art. 9 dieses Protokolls, genannt COSAC (Conférence des Organes spécialisés en Affaires communitaires). ${ }^{69}$

Die COSAC setzt sich nach Ziffer 4.1 ihrer Geschäftsordnung ${ }^{711}$ aus höchstens sechs Mitglicdern des jeweiligen Europaausschusses eines jeden nationalen Parlaments sowie sechs Mitgliedern des Europäischen Parlaments zusammen. Die COSAC dient dem Informations- und Meinungsaustausch zwischen den nationalen Parlamenten und dem Europäischen Parlament und soll die nationalen Parlamente in europapolitischen Angelegenheiten stärken. ${ }^{71}$ Andererseits haben die

69 Wohlard, Bundestag, Bundesrat und Landesparlamente im europäischen Integrationsprozess, 2007, S. 111; Geiger, in: Geiger/Khan/Kotzur, EUV/AEUV, Art. 12 EUV Rn. 9.

70 ABl. 2008, C 27/6.

71 Geiger, in: Geiger/Khan/Kotzur, EUV/AEUV, Art. 12 EUV Rn. 10. 
Beiträge der COSAC keinerlei rechtliche Relevanz. ${ }^{72}$ Nach Art. 10 S. 1 des Protokolls über die Rolle der nationalen Parlamente in der Europäischen Union kann die COSAC ihre Beiträge lediglich zur Kenntnis der europäischen Organe bringen. Nach Art. 10 S. 4 des Protokolls ist eine Bindung der Beiträge gegenüber den nationalen Parlamenten sogar ausdrücklich ausgeschlossen.

Eine weitere Form der interparlamentarischen Zusammenarbeit ermöglicht Art. 125 der Geschäftsordnung des Europäischen Parlaments. ${ }^{73}$ Hiernach kann ein Konvent oder eine Konferenz von Parlamenten einberufen werden, die sich zu zentralen Fragen der EU äußert, wobei die praktischen Erfahrungen mit dieser Einrichtung sehr begrenzt sind. ${ }^{74}$

Zusammenfassend handelt es sich bei der interparlamentarischen Zusammenarbeit nach Art. 12 lit. f EUV primär um eine Form politischer Kooperation ohne rechtliche Wirkungen, deren praktische Bedeutung dadurch keinesfalls in Frage gestellt werden soll.

\section{Legitimation, Konstruktion oder Illusion?}

Durch den Vertrag von Lissabon wird die Mitwirkung der nationalen Parlamente im europäischen Rechtssetzungsprozess erstmals primärrechtlich festgeschrieben. Die nationalen Parlamente können den Rechtssetzungsprozess nicht direkt steuem und werden nicht zu Nebengesetzgebern. Sie erhalten jedoch die Möglichkeit, die Verletzung des Subsidiaritätsprinzips sowie wohl eine Kompetenzüberschreitung der EU zu rügen und deswegen Rechtsschutz einzulegen. Sofern es um die Änderung der primärrechtlichen Grundlagen der EU geht, können die nationalen Parlamente den Prozess insofern „aktiv" gestalten, als sie ihn stoppen können. Wie bei jedem Vetorecht ist es diese destruktive Wirkungsmöglichkeit, die letztlich eine konstruktive Einwirkungsmöglichkeit vermittelt.

Insgesamt mag man insbesondere bei theoretischer Betrachtung deshalb davon ausgehen, dass die demokratische Legitimation stärker auch durch die mitgliedstaatlichen Parlamente vermittelt wird und dadurch eine breitere Basis erhält.

72 Kaufmann-Bühler, in: Lenz/Borchardt, EU-Verträge. Art. 12 EUV Rn. 20; näher Calliess, in: Calliess/Ruffert (Hrsg.), EUV/AEUV, 4. Aufl., Art. 12 Rn. 54.

$73 \mathrm{ABI}$. 2005, L 44/I.

74 Kaufmann-Bühler, in: Lenz/Borchardt, EU-Verträge, Art. 12 EUV Rn. 23; Calliess, in: Calliess/Ruffert (Hrsg.), EUV/AEUV, 4. Aufl., Art. 12 Rn. 58 ff. 
Bei näherer Betrachtung und unter Berücksichtigung der praktischen Parlamentsarbeit mögen allerdings Zweifel angebracht sein. Wenngleich jede Form der Legitimation in gewisser Weise nur eine Konstruktion ist, so mag sich der Glaube an eine zusätzliche über die nationalen Parlamente vermittelte Legitimation möglicherweise als Illusion erweisen. Nur eine Beobachtung und eine Überlegung seien insofern zur Diskussion gestellt:

Zunächst sei auf eine gewisse Widersprüchlichkeit in der Konstruktion des Art. 12 EUV hingewiesen. Während der vor die Klammer der einzelnen Erscheinungsformen gezogene Passus expressis verbis davon spricht, dass die nationalen Parlamente ,aktiv" zur guten Arbeitsweise der Union beitragen, sind die einzelnen Erscheinungsformen zum Teil doch explizit passiv ausgestałtet. Die „aktive" Beteiligung der nationalen Parlamente erschöpft sich in ihrer "passiven" Unterrichtung. Freilich darf der Gegensatz zwischen aktivem Beitragen und bloBem Informiertwerden nicht überbetont werden, weil selbstverständlich ist, dass die hinreichende Unterrichtung notwendige Voraussetzung eines verantwortungsbewussten Handelns der nationalen Parlamente ist. Gleichwohl ist umgekehrt nicht zu übersehen, dass die nationalen Parlamente nach der Konstruktion des Art. 12 eben auch schon dann ,aktiv" zur guten Arbeitsweise der Union beitragen, wenn sie nur passiv Informationen entgegennehmen.

Dies leitet zu der Überlegung über, dass die Konkretisierung der repräsentativen Demokratie durch Aspekte der interparlamentarischen Demokratie in erster Linie eine Konstruktion ist, die zur Illusion einer breiteren und damit besseren demokratischen Legitimation führt. Sieht man einmal von den besonderen Beteiligungsrechten der nationalen Parlamente bei der Entscheidung über Brückenklauseln ab, erschöpfen sich die Mitwirkungsformen im Regelfall der europäischen Rechtsetzung in dem Recht auf Unterrichtung. Der bloße Umstand, dass die nationalen Parlamente informiert sind, genau genormmen sogar der bloße Umstand, dass sie nach Art. 12 lit. a EUV informiert sein sollen, kann aber nicht zu einer höheren demokratischen Legitimation der europäischen Rechtsakte führen. Vielmehr wird hier eine Legitimation nur fingiert, ohne dass es einer ausdrücklichen Willenserklärung der nationalen Parlamente bedarf. Dies mag insofern hingenommen werden, als eben auch nur die normalen europäischen Rechtsetzungsakte betroffer sind und deshalb auch überbaupt kein erhöhter Legitimationsbedarf besteht. Doch gerade mit Blick auf die den nationalen Parlamenten zugedachte maßgebliche Rolle bei der Subsidiatitätskontrolle sind Zweifel angebracht, ob sie dieser Aufgabe gerecht werden können. Dabei mag es vielfältige Gründe dafür geben, dass die nationalen Parlamente und namentlich der Bundestag und der Bundesrat von den Möglichkeiten der Subsidiaritätsrüge oder der Subsidiaritätsklage keinen Gebrauch machen. Inhaltliche Überforderung, fehlen- 
de Sensibilităt oder andere tagesaktuelle Prioritäten mögen hier sehr viel häufiger in Betracht kommen als parteipolitisch motivierter Unwille. Dass die nationalen Parlamente nur ein Akteur unter vielen im Rahmen der Subsidiaritätskontrolle sind, schmälert die Bedeutung einer nicht ausgeübten parlamentarischen Subsidiaritätskontrolle nicht. Denn bei der Subsidiaritätskontrolle geht es mindestens auch, in parlamentarischen Demokratien vielleicht sogar in erster Linie, um die Zuständigkeiten der nationalen Parlamente. Man mag aus einer unterlassenen Subsidiaritätsrüge oder -klage seitens der nationalen Parlamente insofern möglicherweise auf einen Verzicht nationaler Zuständigkeiten schließen, die doch nach der Architektur der Unionsverträge gar nicht zu ihrer Disposition stehen. Doch ob (nationale) Gerichte auf die Klage eines Dritten das Subsidiaritätsprinzip für verletzt erklären, wenn keines der nationalen Parlamente eine Rüge oder Klage erhoben hat, mag bezweifelt werden. Insofern führt die Zuweisung der Subsidiaritätsverantwortung an die nationalen Parlamente möglicherweise zu einer stärkeren Politisierung eines wesentlichen Vertragsgrundsatzes, der an sich doch rechtliche Verbindlichkeit haben soll.

Ähnliche skeptische Betrachtungen ließen sich jedoch für alle Legitimationsformen anstellen. So bleibt die Hoffnung, dass die nationalen Parlamente die ihnen zugewiesenen Befugnisse verantwortungsvoll ausüben, dass sie sich also ihrer Integrationsverantwortung bewusst sind, die sie nicht gegenüber ihrer Regierung. sondern gegenüber den Bürgern haben. Sie, nicht die Parlamente zu stärken, muss das eigentliche Ziel einer interparlamentarischen Demokratie sein. 